\title{
Science Discovery
}

2017; 5(1): 19-24

http://www.sciencepublishinggroup.com/j/sd

doi: $10.11648 /$ j.sd.20170501.14

ISSN: 2331-0642 (Print); ISSN: 2331-0650 (Online)

\section{Distributed Grid-Connected Photovoltaic (pv) Research on the Influence of Power Distribution Network Voltage}

\author{
Yuan An, Hongxin Liu*

\section{Email address:} \\ 407097615@qq.com (Hongxin Liu) \\ ${ }^{*}$ Corresponding author
}

Institute of Water Resources and Hydroelectric Engineering, Xi'an University of Technology, Xi'an, China

\section{To cite this article:}

Yuan An, Hongxin Liu. Distributed Grid-Connected Photovoltaic (pv) Research on the Influence of Power Distribution Network Voltage. Science Discovery. Vol. 5, No. 1, 2017, pp. 19-24. doi: 10.11648/j.sd.20170501.14

Received: January 18, 2017; Accepted: March 22, 2017; Published: March 31, 2017

\begin{abstract}
For the study of distributed grid-connected photovoltaic (pv) affect the quality of power distribution network voltage. Application Matlab respectively different access points in the access of distributed photovoltaic (pv) power distribution network, different capacity and power factor to carry on the simulation. Analysis the influence of distributed photovoltaic access to voltage quality. Results show that the closer it gets to the end of the line network position or and the greater the network capacity, the greater the influence of node voltage photovoltaic system. And the nearer the and node location of nodes affected by the more serious. Because of the photovoltaic system itself will absorb a certain amount of reactive power,so shall be the pv systems with a certain amount of reactive power compensation device. In order to meet the demand for reactive power. Thus the power factor of photovoltaic system is close to 1 .
\end{abstract}

Keywords: Distributed Photovoltaic Power Generation, Simulation Analysis, Voltage Quality

\section{分布式光伏发电并网对配电网电压质量影响的研究}

安源, 刘宏欣 ${ }^{*}$

水利水电学院, 西安理工大学, 西安, 中国

邮箱

407097615@qq. com（刘宏欣）

摘要: 为研究分布式光伏发电并网对配电网电压质量影响, 应用Matlab分别对接入分布式光伏的配电网中不同接入点、 不同容量和不同功率因数进行仿真模拟, 分析了分布式光伏接入对电压质量的影响。结果表明: 并网点位置越接近线 路末端或者并网点容量越大, 光伏系统对节点的电压影响越大, 而且离并网点位置越近的节点所受影响越严重。由于 光伏系统自身也会吸收一定的无功, 所以应为光伏系统配备一定量的无功补偿装置, 以满足起对无功的需求, 从而光 伏系统的功率因数接近 1 。

关键词: 分布式光伏发电, 仿真分析, 电压质量 


\section{1. 引言}

太阳能光伏发电在 21 世纪会占据世界能源消费的重 要席位, 不但要代替部分常规能源, 而且将成为世界能源 供应的主体。在当今油、碳短缺的现状下, 各国都加紧了 光伏的步伐, 在发展低碳经济的大背景下, 各国政府对光 伏发电的认可度逐渐提高。为改善能源结构, 更加充分利 用分布式清洁能源, 国家电网再次颁布了《关于做好分布 式电源并网服务的工作意见》, 这是继国家启动分布式光 伏发电支持政策的又一力作 [1]。这些政策的颁布旨在鼓 励分布式光伏电源 (Photovoltaic Distributed Generation, PV-DG) 等分布式电源并入电网运行, 但其 接入电网, 首当其冲的改变了配电网的潮流方向, 这种潮 流的变化影响着配电网的电压分布 [2]。一方面, 分布式 光伏的合理配置会对配电网的电压具有支撑作用, 另一方 面分布式光伏的无约束运行可能导致配电网某些节点出 现过电压或者严重的电压波动 [3]。本文主要分析分布式 光伏并网对配电网电压的影响, 通过使用Matlab软件搭建 配电网、负荷模型, 仿真分析分布式光伏电源接入不同容 量、不同位置、不同功率因数及多个分布式光伏电源接入 等对配电网电压的影响规律。

\section{2. 太阳能光伏发电原理及组成}

\section{1. 太阳能光伏发电原理}

光伏发电是利用半导体界面的光生伏特效应 [4]而将 光能直接转变为电能的一种技术。主要由太阳电池板 (组 件)、控制器和逆变器三大部分组成, 主要部件由电子元 器件构成。太阳能电池经过串联后进行封装保护可形成大 面积的太阳电池组件, 再配合上功率控制器等部件就形成 了光伏发电装置。
太阳能是一种辐射能, 它必须借助于能量转换器件才 能变换为电能。这种把辐射能变换成电能的能量转换器件, 就是太阳能电池。太阳能电池是利用光电转换原理使太阳 的辐射光通过半导体物质转变为电能的器件, 这种光电转 换过程通常叫做 “光生伏打效应”, 太阳能电池又称为 “光 伏电池”。当太阳光照射到由 $\mathrm{P} 、 \mathrm{~N}$ 型两种不同导电类型的 同质半导体材料构成的 $\mathrm{P}-\mathrm{N}$ 结上时, 在一定条件下, 太阳 能辐射被半导体材料吸收. 形成内建静电场。如果从内建 静电场的两侧引出电极并接上适当负载, 就会形成电流, 这就是太阳能电池的基本原理。单片太阳能电池就是一薄 片半导体 $\mathrm{P}-\mathrm{N}$ 结。标准光照条件下, 额定输出电压为 $0.48 \mathrm{~V}$ 。 为了获得较高的输出电压和较大容量, 往往把多片太阳能 电池连接在一起, 目前, 太阳能电池的光电转换率一般在 $15 \%$ 左右, 个别发达国家的实验室太阳能电池光电转换率 已经可以达到 $30 \%$ 左右。

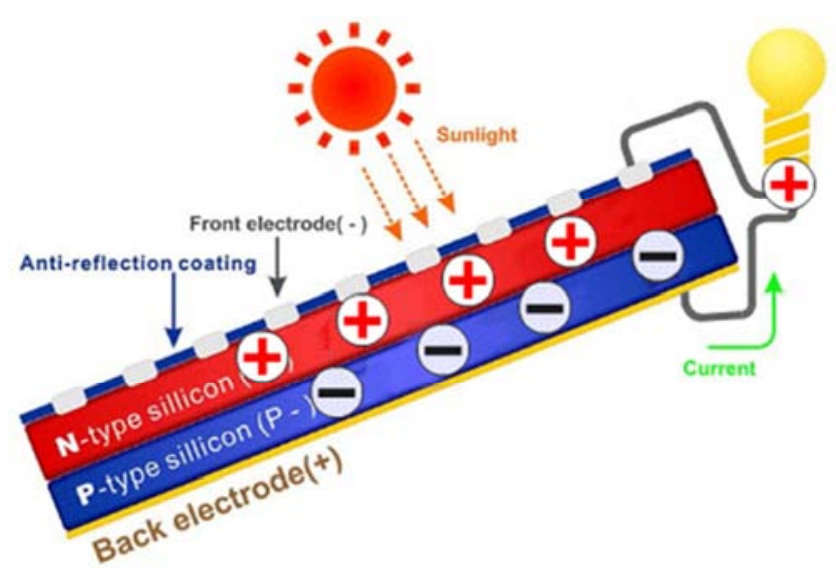

图1 太阳能发电原理图。

太阳能光伏电站主要由太阳电池板 (组件) 、控制器 和逆变器三大部分组成, 如图2所示。

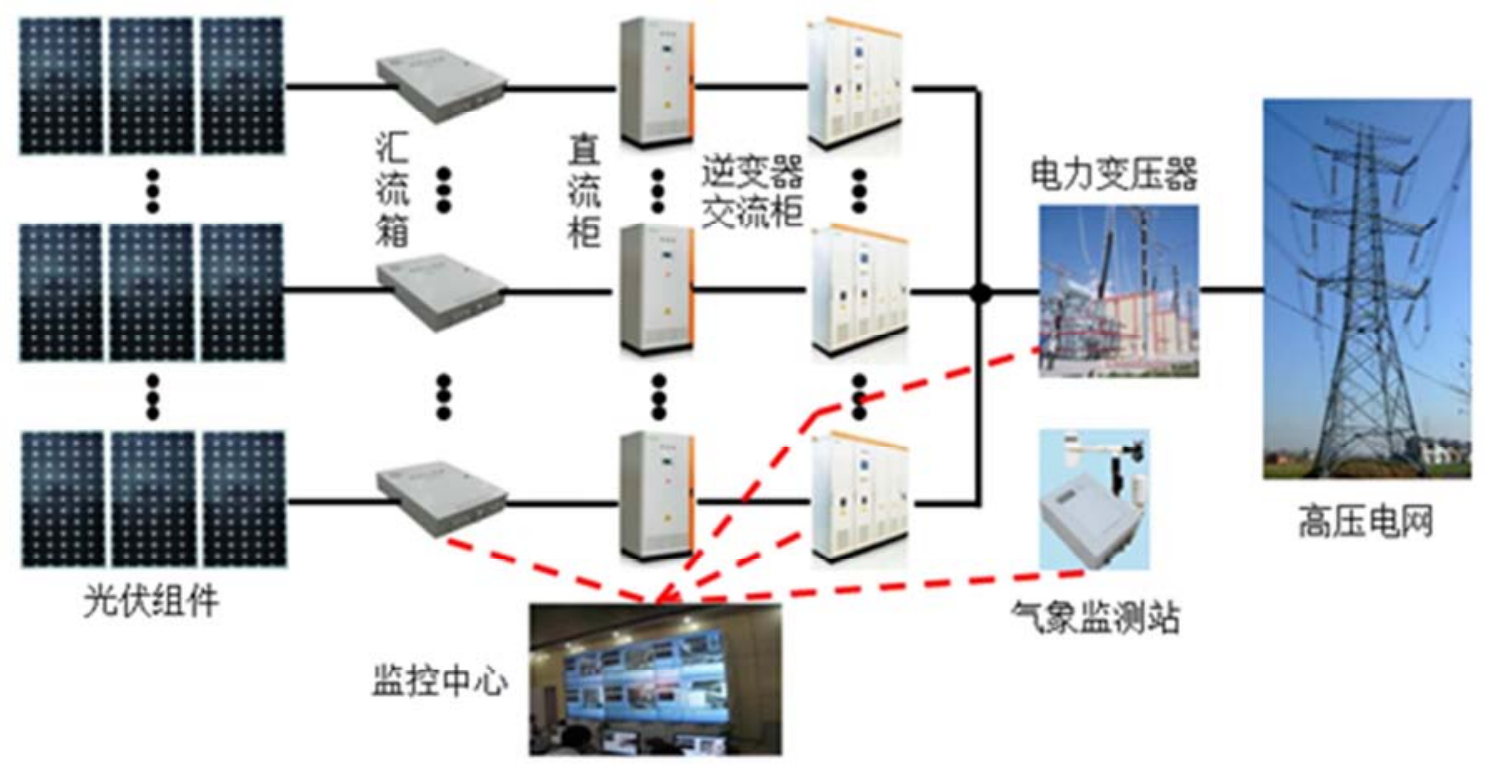

图2 光伏电站系统图。 


\section{2. 太阳能光伏电站的基本结构}

光伏发电系统主要由太阳能电池阵列、逆变器及并网 系统三大部分组成, 其中太阳电池阵列及逆变器组合为发 电单元部分。

\subsection{1. 太阳能电池}

太阳能电池是利用光伏效应将太阳能直接转换成电 能的装置。当N型和P型两种不同型号的半导体材料接触后, 由于扩散和漂移作用, 在界面处形成由P型指向N型的内建 电场。太阳能电池吸收一定能量的光子后, 半导体内部产 生电子一空穴对, 电子带负电, 空穴带正电。在 $\mathrm{P}-\mathrm{N}$ 结内 建电场的作用下, 电子和空穴被分离, 产生定向运动, 并 被太阳能电池的正、负极收集, 在外电路中产生电流, 从 而获得电能。

\subsection{2. 逆变器}

集中式逆变器顾名思义是将光伏组件产生的直流电 汇总转变为交流电后进行升压、并网。因此, 逆变器的功 率都相对较大。光伏电站中一般采用 $500 \mathrm{~kW}$ 以上的集中式 逆变器。

组串式逆变器顾名思义是将光伏组件产生的直流电 直接转变为交流电汇总后升压、并网。因此, 逆变器的功 率都相对较小。光伏电站中一般采用 $50 \mathrm{~kW}$ 以下的组串式逆 变器。[5]

\section{3. 太阳能光伏电站分类}

根据太阳能光伏电站建设安装方式及规模, 可将其分 为两类: 集中式光伏电站和分布式光伏电站。

（一）集中式光伏电站：充分利用荒漠地区丰富和相 对稳定的太阳能资源构建大型光伏电站, 接入高压输电系 统供给远距离负荷。在集中式并网光伏电站中，太阳能通 过太阳能电池组成的光伏组件方阵转换成直流电, 经过三 相逆变器 (DC-AC) 转换成电压较低的三相交流电, 再通 过升压变压器转换成符合公共电网电压要求的交流电, 并 直接接入公共电网, 供公共电网用电设备使用和远程调配。

优点: (1) 由于选址更加灵活, 光伏出力稳定性有 所增加, 并且充分利用太阳辐射与用电负荷的正调峰特性, 起到削峰的作用。（2）运行方式较为灵活, 相对于分布
式光伏可以更方便地进行无功和电压控制, 参加电网频率 调节也更容易实现。（3）建设周期短, 环境适应能了强, 不需要水源、燃煤运输等原料保障, 运行成本低, 便于集 中管理, 受到空间的限制小, 可以很容易地实现扩容。缺 点:（1）需要依赖长距离输电线路送电入网, 同时自身 也是电网的一个较大的干扰源, 输电线路的损耗、电压跌 落、无功补偿等问题将会凸显。（2）大容量的光伏电站 由多台变换装置组合实现, 这些设备的协同工作需要进行 同一管理, 目前这方面技术尚不成熟。(3) 为保证电网 安全, 大容量的集中式光伏接入需要有LVRT等新的功能, 这一技术往往与孤岛存在冲突。

（二）分布式光伏电站：利用分散式资源，装机规模 较小的、布置在用户附近的发电系统, 它一般接入低于 35 千伏或更低电压等级的电网。主要基于建筑物表面, 就近 解决用户的用电问题, 通过并网实现供电差额的补偿与外 送。优点: (1) 光伏电源处于用户侧, 发电供给当地负 荷, 视作负载, 可以有效减少对电网供电的依赖, 减少线 路损耗。（2）充分利用建筑物表面, 可以将光伏电池同 时作为建筑材料, 有效减少光伏电站的占地面积。3、与 智能电网和微电网的有效接口, 运行灵活, 适当条件下可 以脱稿电网独立运行。缺点:（1）配电网中的潮流方向 会适时变化, 逆潮流导致额外损耗, 相关的保护都需要重 新整定, 变压器分接头需要不断变换, 等问题。(2) 电 压和无功调节的困难, 大容量光伏的接入后功率因数的控 制存在技术型难题, 短路电力也将增大。(3) 需要在配 电网级的能量管理系统, 在大规模光伏接入的情况下进行 负载的同一管理。对二次设备和通讯提供了新的要求, 增 加了系统的复杂性。

\section{4. 分布式光伏电源对配电网电压影响的理论分 析}

图1所示为PV接入典型放射状配电网10KV的负荷分布 [6], 线路共有负荷电 $\mathrm{N}$ 个, 第 $\mathrm{n}$ 个复合点消耗功率为 $P_{n}+j Q_{n}(n=1,2 \ldots N)$, 分布式光伏电源 $\mathrm{DG}$ 接入容量为 $P_{D G}+j Q_{D G}$, 假设 $\mathrm{DG}$ 接入点为 $\mathrm{m}$, 第 $\mathrm{n}-1$ 和第 $\mathrm{n}$ 个负荷点之间 的线路阻抗为 $R_{n}+j X_{n}(n=1,2 \ldots N)$ 。定义潮流向负载方向 流动为正, 反之为负, 线路损耗忽略不计。

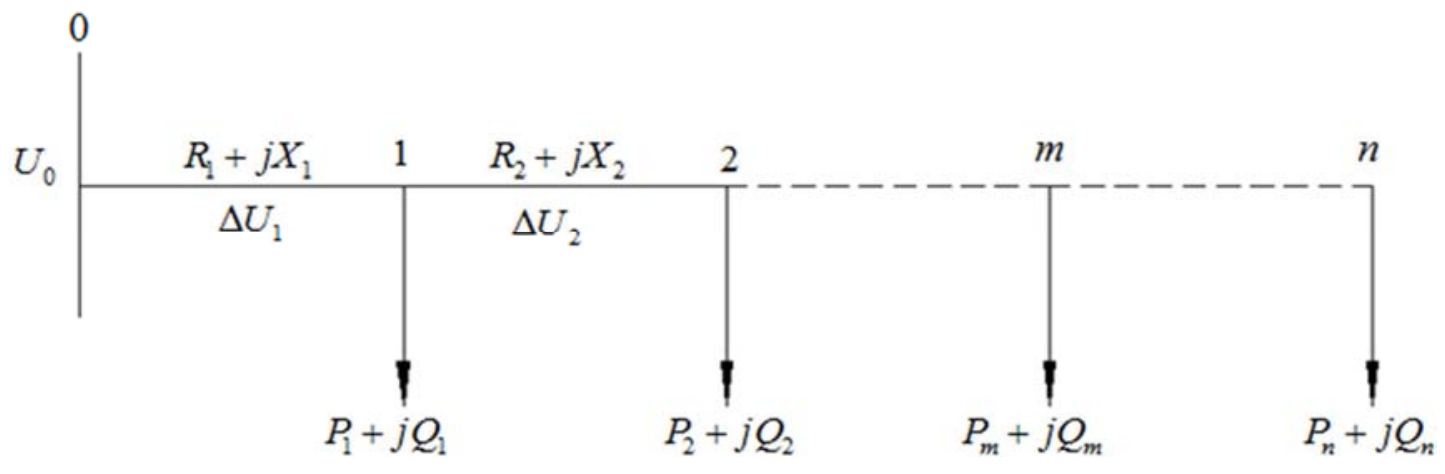

图3 PV接入典型放射状配电网10KV的负荷分布。 
(一) $\mathrm{DG}$ 接入前,

两点间电压降

$$
\Delta U_{n}=U_{n-1}-U_{n}=\frac{R_{n} \sum_{i=n}^{N} P_{i}+X_{n} \sum_{i=n}^{N} Q_{i}}{U_{n-1}}
$$

任一节点 $n$ 的电压

$$
U_{n}=U_{0}-\sum_{j=1}^{N} \Delta U_{n}=U_{0}-\sum_{j=1}^{N} \frac{R_{j} \sum_{i=j}^{N} P_{i}+X_{j} \sum_{i=j}^{N} Q_{i}}{U_{j-1}}
$$

式 (1) 和 (2) 中用户小号的有功功率 $P_{i}$ 和无功功率 $Q_{i}$ 都大于 0 , 故节点 $\mathrm{n}-1$ 和节点 $\mathrm{n}$ 间之的电压差都大于 0 , 且 馈线上各点的电压都低于母线电压并随着与母线距离的 增加而降低。为满足馈线末端的电压满足电压偏差的规定, 一般采用提高馈线首端电压和增加无功补偿设备的方法 [7]。

（二） $\mathrm{DG}$ 接入后, 当在节点 $\mathrm{m}$ 上接入分布式光伏电源 $D G$, 如图2所示。

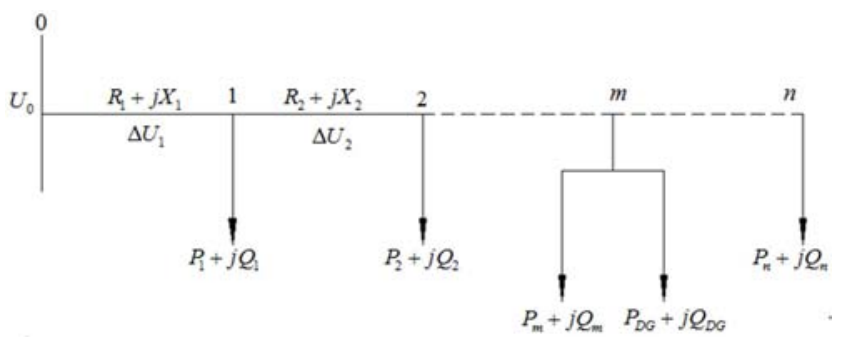

图4 DG接入后的负荷分布。

(1)点 $m$ 接入 $D G$, 相应的节点 $m$ 电压为

$$
U_{m}^{\prime}=U_{0}-\sum_{j=1}^{m} \frac{R_{j}\left(\sum_{i=j}^{N} P_{i}-P_{D G}\right)+X_{j}\left(\sum_{i=j}^{N} Q_{i}-Q_{D G}\right)}{U_{j-1}^{\prime}}>U_{m}
$$

比较 (2) 和 (3) , 接入 $D G$ 后, 接入光伏的 $m$ 点电压 升高, 且变化为 $\sum_{j=1}^{m} \frac{P_{D G} R_{j}+Q_{D G} X_{j}}{U_{j-1}}$ 。

(2) $D G$ 接入后, 若负荷点 $\mathrm{P}$ 位于光伏接入点 $\mathrm{m}$ 之前 $(0<\mathrm{P}<\mathrm{m})$,

$[1]$ 则 $P$ 点电压为:

$$
U_{p}^{\prime}=U_{0}-\sum_{j=1}^{p} \frac{R_{j}\left(\sum_{i=j}^{N} P_{i}-P_{D G}\right)+X_{j}\left(\sum_{i=j}^{N} Q_{i}-Q_{D G}\right)}{U_{j-1}}>U_{p}
$$

$\mathrm{GD}$ 接入后可以起到提升电压的作用, 在线路参数、用 户负荷大小确定时, 电压提升的幅度与光伏电源出力、接 入位置密切相关。

[2]相邻两点之间的电压差为

$$
\Delta U_{p}^{\prime}=U_{p-1}^{\prime}-U_{p}^{\prime}=\frac{R_{p}\left(\sum_{i=p}^{N} P_{i}-P_{D G}\right)+X_{p}\left(\sum_{i=p}^{N} X_{i}-X_{D G}\right)}{U_{p-1}^{\prime}}
$$

若忽略用户线路的小电抗和无功影响:

$$
\Delta U_{p}^{\prime}=U_{p-1}^{\prime}-U_{p}^{\prime}=\frac{R_{p}\left(\sum_{i=p}^{N} P_{i}-P_{D G}\right)}{U_{p-1}^{\prime}}
$$

对比（1）和（6）, 得出 $\Delta U_{p}^{\prime}<\Delta U_{p}$ 说明接入后节点 $\mathrm{p}-1$ 和节点 $\mathrm{p}$ 之间的压降小于未接入 $D G$ 时的压降。当接入 $D G$ 时, 其接入容量的大小对 $\mathrm{p}$ 节点电压有影响: 当 $\sum_{i=p}^{N} P_{i}>P_{D G}$ 时, $\Delta U_{p}^{\prime}>0$, 所以 $\mathrm{p}-1$ 节点电压大于 $\mathrm{p}$ 节点电压, 电压降 低; 当 $\sum_{i=p}^{N} P_{i}<P_{D G}$ 时, $\Delta U_{p}^{\prime}<0$, 所以 $\mathrm{p}-1$ 节点电压小于 $\mathrm{p}$ 节点电压, 电压升高;

(3) $D G$ 接入后, 若负荷点 $\mathrm{P}$ 位于光伏接入点 $\mathrm{m}$ 之后 $(\mathrm{m}<\mathrm{P}<\mathrm{N})$,

[1]则 $P$ 点电压为: (忽略无功和电抗影响)

$$
U_{p}^{\prime}=U_{0}-\sum_{j=1}^{m} \frac{R_{j}\left(\sum_{i=j}^{N} P_{i}-P_{D G}\right)}{U_{j-1}}-\sum_{j=m+1}^{p} \frac{R_{j} \sum_{i=j}^{N} P_{i}}{U_{j-1}}
$$

[2]相邻两点之间的电压降:

$$
\Delta U_{p}^{\prime}=U_{p-1}^{\prime}-U_{p}^{\prime}=\sum_{j=m+1}^{p} \frac{R_{j} \sum_{i=j}^{N} P_{i}}{U_{j-1}}-\sum_{j=m+1}^{p-1} \frac{R_{j} \sum_{i=j}^{N} P_{i}}{U_{j-1}}>0
$$

由式 (8) 可知, 第 $\mathrm{p}-1$ 点电压始终大于第p点, 电压 呈将低趋势。

从以上的分析可以得知, 当单一 $D G$ 接入后, $D G$ 的容量、 接入位置、功率因数这几个因素将会使有关节点的电压产 生变化。多个分布式光伏电源接入的情况可按照相同的方 法计算 [8]。

\section{5. 模型搭建和仿真分析}

\section{1. 搭建模型}

以 $10 \mathrm{kV}$ 的均匀配电网为例, 线路长度 $12 \mathrm{~km}$, 配电网负 荷为 $S=P+Q=8+j 3.6$ (MVA)。线路单位阻抗为 $Z=0.08+j 0.25$ $(\Omega)$, 设置节点 $1-6$, 间距均为 $2 \mathrm{~km}$ 。功率和电压基准值 分别为 $S_{B}=10 \mathrm{MVA} 、 U_{B}=10 \mathrm{KV}$ 。线路首端电压标么值 $U_{0}=1.05$ 和馈线额定电压标么值 $U_{N}=1.0$ 。 


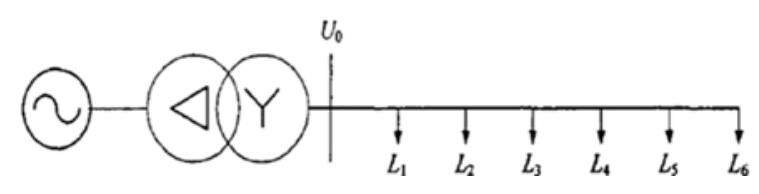

图5 配电网结构图。

\section{2. 仿真分析}

\subsection{1. 光伏电源接入位置对电压质量影响}

对单个同容量PV, 改变其在线路中的位置进行仿真。 把相同容量PV分别接入节点 1 至 6 处。若PV容量为4MVA, 功 率因数为 0.9 (滞后)。所得电压分布图如下:

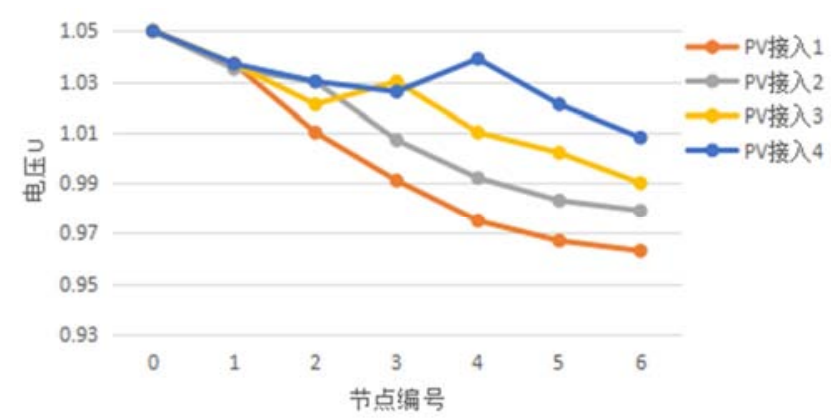

图6 基于不同接入位置的电压分布图。

PV接入系统, 会使馈线电压发生明显变化。与不接入 DG的情况相比, PV对节点确实起到了支撑电压的作用, 且 在容量不变的情况下, 接入点未知的变化, 电压分布也随 之发生变化。DG接入点越接近母线, 对馈线电压分布的影 响越小; DG接入点越接近馈线末端, 对整个线路电压分布 的影响越大。所以选择在末端并网有助于对整体电压的提 升。但是要注意在末端并网时PV容量的选择, 要保证接入 PV后，各节点不超过安全电压 $[9]$ 。

\subsection{2. 光伏电源接入容量对电压质量影响}

仅改变PV容量, 固定PV接入点位置, 在固定位置节点 3 和节点 5 分别引入容量为 $S=2 M V, S=3 M V, S=4 M V$, 且PV功 率因数为 0.9 (滞后)。所得电压分布图如下

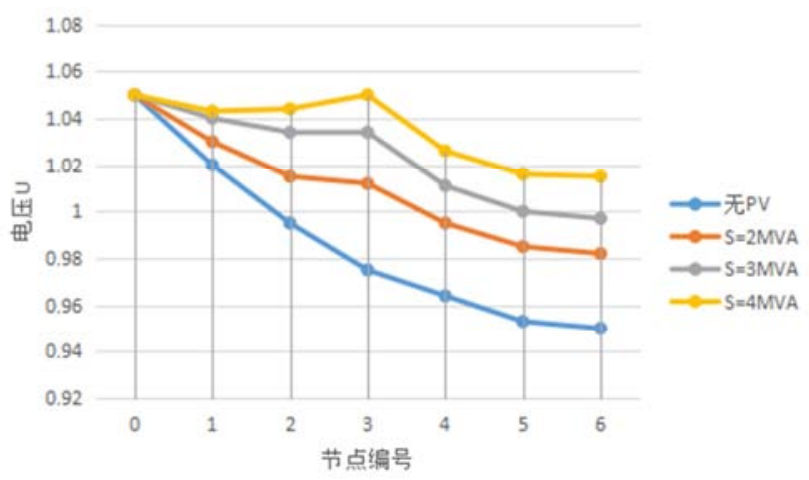

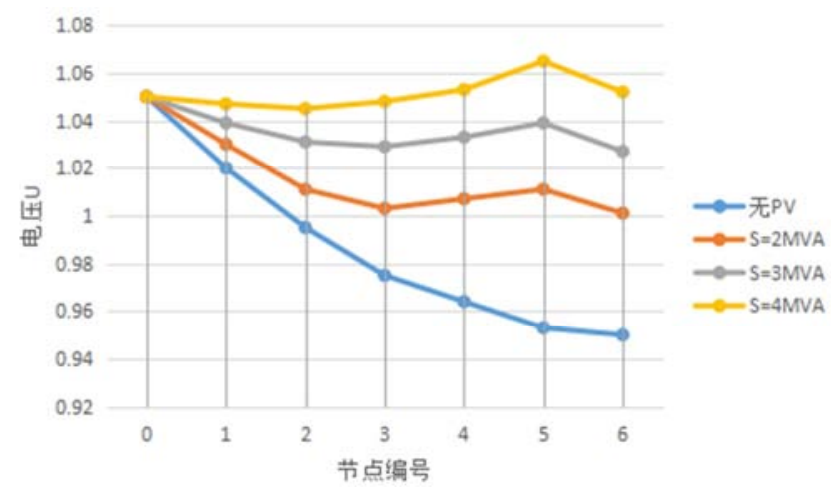

图7 基于不同接入容量的电压分布图。

在不改变PV引入位置时, 不同容量的DG接入, 对馈线 电压影响不同。接入DG后, 由于减少了馈线中的传输功率, 馈线的节点电压整体提高。且随着 $D G$ 容量的增加, 整条馈 线的电压水平都在提高, 接入点甚至有可能超过电压允许 的偏差范围 $[10]$ 。

\subsection{3. 光伏电源的功率因数对电压质量影响}

在保持DG的接入容量、位置不变的情况下，仅仅通过 改变功率因数来比较不同功率因数的DG接入对馈线电压 的影响。固定PV接入点在节点 2 , 功率因数分别取滞后 0.8 、 滞后0.9、1、超前 0.8 、超前 0.9 。 [11]

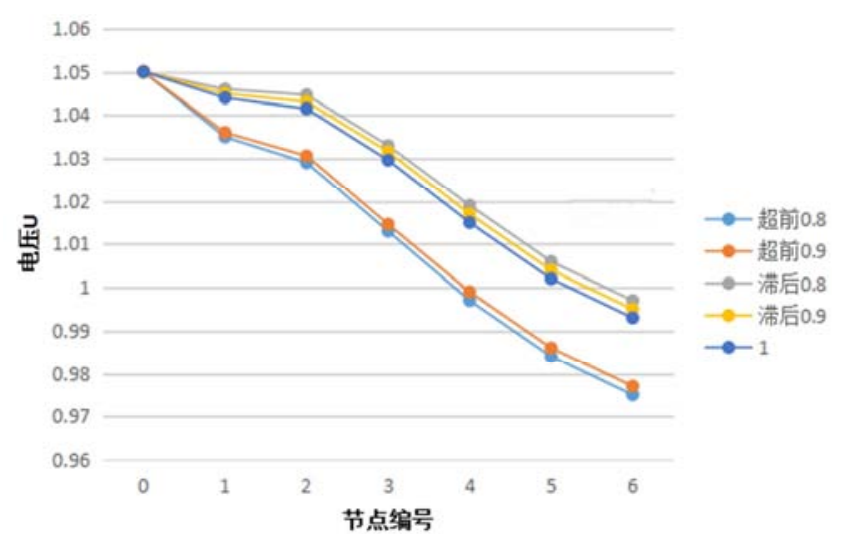

图8 基于不同功率因素的电压分布图。

不同功率因数DG的接入对馈线电压会产生不同的影 响。就改善馈线电压来看, 接入滞后功率因数的DG较接入 超前功率因数的DG效果更好。当滞后功率因数的DG接入配 电网时, DG不仅发出有功, 同时发出无功, 所以对馈线电 压支持更强 [12]。而超前功率因数DG接入时, 发出的有功 减少了有功使电压升高, 但是同时吸收无功使电压降低, 因而最终的DG接入点的电压变化取决于有功和无功这两 方面的和 [13]。

\section{6. 结论}

模拟配电网模型, 通过Matlab进行仿真, 分析了分布 式光伏并入配电网对配电网电压质量的影响, 得到以下几 点结论: 
（1）当分布式光伏系统接入配电网系统，对影响配 电网电压的因数有分布式光伏的接入点位置、容量和功率 因数等。

（2）并网点位置越接近线路末端或者并网点容量越 大, 光伏系统对节点的电压影响越大, 而且离并网点位置 越近的节点所受影响越严重。

（3）由于光伏系统自身也会吸收一定的无功，所以 应为光伏系统配备一定量的无功补偿装置, 以满足起对无 功的需求, 从而光伏系统的功率因数接近 1 。

\section{参考文献}

[1］国家电网公司.《关于做好分布式电源并网服务的工作意见》 [EB/OL]。 [2013-02-27]。

[2] 武晓朦, 刘健, 毕鹏翔等. 配电网电压稳定性研究 [J]. 电网 术, 2006, 30 (24) : 31-35.

D0I: 10.3321/j. issn:1000-3673. 2006.24.007。

[3] 张吉, 李庚银, 魏军强等. 考虑分布式电源随机特性的配电 网电压质量概率评估 $[\mathrm{J}]$. 中国电机工程学 报, 2013, 33 (13) : 150-156, 前插18.

[4] 芦浩. 太阳能光伏发电技术应用研究与普及 $[J]$ 中国新技术 新产品, 2016, (16)。

[5] 周京华, 刘劲东, 陈亚爱, 李津等. 大功率光伏逆变器的低电 压穿越控制 [J]. 电网技术2013, (07)。
[6] 彭灵. 多电源下配电网继电保护若干问题的探讨 $[J]$. 电气 关, 2015, 53(4): 9-11. D0I : 10. 3969/j. issn. 1004-289X. 2015. 04. 003 。

[7] 黄巍, 吴俊勇, 鲁思棋等. 电压偏差和谐波约束下配网光伏 最大渗透率评估 $[\mathrm{J}]$. 电力系统保护与控 制, 2016, 44 (6) :49-55. D0I : 10.7667/PSPC150842。

[8］栗然, 马慧卓, 祝晋尧等. 分布式电源接入配电网多目标优 化规划 $[J]$. 电力自动化设备, 2014,34(1):6-13. DOI: 10. 3969/j. issn. 1006-6047.2014.01.002。

[9] Hiyama T, Zuo D, Funabashi T. Multi-agent based control and operation of distribution system with dispersed power source $[J]$. Proc. of IEEE Power Engineering Society Transmission and Distribution Conference. Asia pacific, 2012, 2129²133.

[10] Naresh Acharya, Pukar Mahat, N. Mithulanantha. An analytical approa ch for DG allocation in primary distribution network $[\mathrm{J}]$. Electrical Power and Energy ystems, 2006, 28(2):669-678.

[11] 吕清洁, 王韶, 刘庭否等. 含分布式发电的配电网有功-无功 综合优化 $[\mathrm{J}]$. 电力系统保护与控

制, 2012, 40 (10): 71-76, 83.

DOI: 10. 3969/j. issn. 1674-3415. 2012. 10.013。

[12] 周念成, 楼晓轩, 王强钢等. 电网电压不平衡下三项光伏发 电系统的谐波电流抑制 $[\mathrm{J}]$. 电工技术学 报, 2015, 30(16) :246-254。

[13] 崔红芬, 汪春, 叶季蕾等. 多接入点分布式光伏发电系统与 配电网交互影响研究 $[\mathrm{J}]$. 电力系统保护与控制, 2015, (10)。 